\title{
Complete response to abdominal bulky lymph node recurrence in an esophageal cancer patient treated with S-1 monotherapy: A case report
}

\author{
ATSUTO KATANO, HIDEOMI YAMASHITA, KAE OKUMA and KEIICHI NAKAGAWA \\ Department of Radiation Oncology, University of Tokyo Hospital, Tokyo 113-8655, Japan
}

Received March 17, 2015; Accepted April 1, 2016

DOI: $10.3892 / 01.2016 .4462$

\begin{abstract}
Esophageal cancer is a highly lethal malignancy of the upper gastrointestinal tract. The recurrence of the cancer indicates a poor prognosis, and even salvage therapy using multi-agent combination chemotherapy, which is considered more effective than single-agent chemotherapy, is not able to achieve a sufficient response. The present study reports the case of a 74-year-old male who presented to a local hospital with dysphagia in February 2006. Upon radiographic and pathological examination, the patient was diagnosed with squamous cell carcinoma of esophagus that was clinically staged as T3N1M1. The patient was referred to the University of Tokyo Hospital (Tokyo, Japan) for concurrent chemoradiotherapy using a radiation dose of 50.4 Gy in 28 fractions, and subsequently achieved a complete response (CR). At 8 years after the initial diagnosis and subsequent treatment, the patient presented with abdominal swollen lymph nodes that were $38 \mathrm{~mm}$ and $22 \mathrm{~mm}$ in diameter. Recurrent metastasis was diagnosed. A single-agent regimen was selected due to the patient's poor performance status. This consisted of 2 cycles of oral S-1 administration twice a day for 2 consecutive weeks, followed by 2 weeks of rest ( $80 \mathrm{mg}$ /day for 14 days/cycle). A CR was achieved following the use of S-1 administration as salvage therapy. The patient exhibited no signs of recurrence subsequent to 9 months of follow-up. Overall, the present study reports that $\mathrm{S}-1$ administration shows marked effectiveness in the treatment of huge recurrent lesions. S-1 is considered as a good treatment option in patients with poor a performance status who require salvage therapy.
\end{abstract}

Correspondence to: Mr. Atsuto Katano, Department of Radiation Oncology, University of Tokyo Hospital, 7-3-1 Hongo, Bunkyo-ku, Tokyo 113-8655, Japan

E-mail: atsuto-katano@umin.ac.jp

Abbreviations: 5-FU, 5-fluorouracil; CDDP, cisplatin; CR, complete response; $\mathrm{CT}$, computed tomography; GIF, gastrointestinal fiberscopy

Key words: esophageal cancer, salvage therapy, S-1, recurrence, squamous cell carcinoma

\section{Introduction}

Esophageal cancer affects $>45$ million people worldwide, and the incidence is rapidly increasing (1). The 5-year survival rate for patients with esophageal cancer has gradually improved to $\sim 20 \%$ in the past 30 years (2), however, the disease still has a poor prognosis. Since early-stage esophageal cancer has few symptoms, the cancer is often found in the locally advanced or metastatic state, and $\sim 40 \%$ of patients have distant metastatic lesions at the time of presentation (3). Moreover, despite adequate initial treatment, patients remain at risk of developing recurrence.

Recurrence often occurs at the lymph nodes, bones, lungs and liver (4). Treatment of recurrent esophageal cancer depends on the initial treatment, the histology of the primary tumor and the location of the recurrence. The salvage therapy for recurrent esophageal cancer is aimed at providing a survival benefit or improving the patient's quality of life, and it rarely results in complete remission.

Combination chemotherapy is considered to be more effective than single-agent chemotherapy, but has more toxic effects for the patient (5). The regimen for salvage therapy remains controversial. In the present study, due to the condition of the patient, S-1 administration was selected as salvage chemotherapy and greater than expected effectiveness was observed.

\section{Case report}

A 74-year-old male presented to the Department of Pathology, Sanikukai Hospital (Tokyo, Japan), in February 2006 after experiencing dysphagia and weight loss of $5 \mathrm{~kg}$ over the preceding 6 months. The patient drank $360 \mathrm{ml}$ of rice wine twice a week, and had smoked 15 cigarettes per day for the past 55 years. Gastrointestinal fibroscopy (GIF) and computed tomography (CT) scans were performed. GIF revealed a 3-4-cm Borrmann type 2 lesion covering $~$ one-third of the circumference of the esophagus, $35 \mathrm{~cm}$ from the incisor teeth. The lesion was biopsied and diagnosed as squamous cell carcinoma by examination of the pathological specimen. CT scans show showed enlarged lymph nodes around the lesser curvature of the stomach at the cardiac end. The patient was clinically staged as T3N1M0, according to the 6th edition of the American Joint Committee on Cancer tumor-node-metastasis 
staging system (6). Surgery was not recommended due to the age of the patient.

The patient was referred to the University of Tokyo Hospital (Tokyo, Japan) for chemoradiotherapy on April 2006. Positron emission tomography CT imaging revealed primary lesions in the lower esophageal region and in the cardiac end of the stomach. Further CT scans revealed a $60-\mathrm{mm}$ dorsal dominant lesion with circumferential wall thickening that was infiltrating the proper muscular layer in the mid and lower thoracic esophagus. A 70x50-mm enhanced lymph node was noted infiltrating the proper muscular layer at the cardiac end of the stomach, and an enlarged lymph node was present around the left renal vein. These findings were compatible with the clinical stage, T3N1M1. The patient was administered 2 cycles of chemotherapy (28 days/cycle) comprising a combination of cisplatin (CDDP; $75 \mathrm{mg} / \mathrm{m}^{2}$ ) on day 1 and 5 -fluorouracil (5-FU; $1,000 \mathrm{mg} / \mathrm{m}^{2}$ ) on days $1-4$. The patient was concurrently administered radiotherapy, with a clinical target volume that included the whole esophageal tract, para-esophageal lymph nodes, lesser curvature lymph nodes and para-aortic lymph nodes. A $10 \mathrm{MV}$ X-ray parallel-opposed pair 4-field oblique box and 50.4 Gy in 1.8-Gy daily fractions were used. Acute adverse events, including grade 2 dermatitis, grade 4 anemia, grade 4 leukopenia and grade 3 thrombocytopenia, as assessed by the Common Terminology Criteria for Adverse Events (version 3.0) (7), were noted; granulocyte colony-stimulating factor administration and a transfusion of 4 units of red blood cell concentrate in mannitol adenine phosphate and 10 units of platelet concentrate were subsequently required. Following the concurrent chemoradiotherapy, CT scans showed that the thickening of the esophageal wall was reduced and that the lymph nodes at the cardiac end of the stomach and para-aorta were markedly reduced in volume. GIF revealed no clear abnormalities, with the exception of a scar-like lesion on the right wall of the esophagus, $32-38 \mathrm{~cm}$ from the incisor teeth. Accordingly, this treatment was noted to have resulted in a complete response (CR). Follow-up examinations included a physical examination, the assessment of laboratory data, GIF and CT scans.

The patient showed no signs of recurrence after the initial treatment, however, in December 2013, the patient experienced dysphagia and a CT scan (Aquilion LB; Toshiba Medical Systems Corporation, Tokyo, Japan) showed a swollen lymph node around the common hepatic artery. In March 2014, the lymph node around the common hepatic artery had increased to $38 \mathrm{~mm}$ in diameter and the sub-pyloric lymph node had increased to $22 \mathrm{~mm}$ in diameter (Fig. 1); this was diagnosed as metastatic recurrence. The patient decided to receive salvage chemotherapy consisting of S-1 monotherapy following consideration of the diminished renal function, which may have been caused by initial treatment of CDDP administration. The patient's serum creatinine level was $1.49 \mathrm{mg} / \mathrm{dl}$ and the blood urea nitrogen level was $29.7 \mathrm{mg} / \mathrm{dl}$. S-1 was administered twice a day during a 2 -week period, followed by a 2 -week rest period ( $80 \mathrm{mg} /$ day for 14 days/cycle). After 2 cycles of S-1 monotherapy, CT scans (Aquilion LB; Toshiba Medical Systems Corporation) showed that the existing lymph node lesions in the abdominal area were markedly reduced in volume. Follow-up examinations included a physical examination, assessment of tumor markers and CT scans. The patient

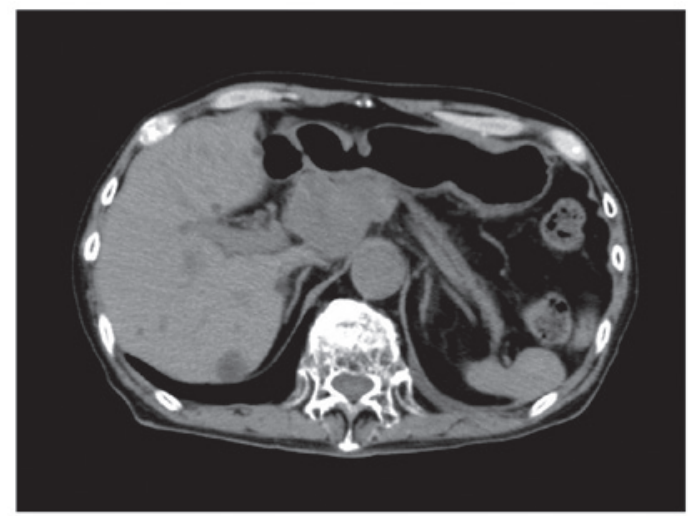

Figure 1. Abdominal computed tomography performed in March 2014, showing that the lymph node around the common hepatic artery had increased to $38 \mathrm{~mm}$ in diameter and that the sub-pyloric lymph node had increased to $22 \mathrm{~mm}$ in diameter.

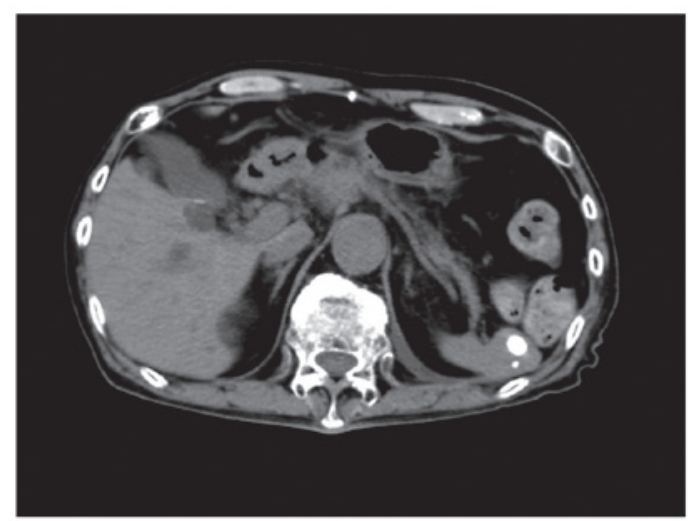

Figure 2. Abdominal computed tomography performed in March 2015 at 9 months after S-1 monotherapy showing no enlarged lymph node lesions.

achieved a CR and has exhibited no signs of recurrence for 9 months (Fig. 2). However, in June 2015 a CT scan revealed multiple lymphadenopathy of the abdominal lymph nodes. The patient was subsequently administered chemotherapy with tegafur (300 mg/kg, daily) for 2 months, but succumbed due to progressive disease in December 2015.

\section{Discussion}

There is no consensus as to the best regimen for the chemotherapeutic treatment of advanced, recurrent or metastatic esophageal cancer. The active single agents for squamous cell carcinoma are CDDP, 5-FU, bleomycin, paclitaxel, mitomycin, mitoguazone, vinorelbine and methotrexate. Combination chemotherapy with CDDP is widely used for esophageal cancer treatment. The response rate of targeted CDDP/5-FU treatment for advanced and recurrent cases of squamous cell carcinoma has been recorded at 36\% (8). Whereas two-drug regimens are preferred due to a lower toxicity, three-drug regimens are adopted for more medically fit patients with a good performance status. Combination treatment with CDDP and fluoropyrimidine is regarded as first-line therapy for esophageal and esophagogastric junction cancers (9). For advanced esophagogastric cancer, combination chemotherapy with 
epirubicin plus CDDP/5-FU has shown a good response rate of $40.7 \%$, a 1-year survival rate of $37.7 \%$ and a median survival time of 9.9 months (10).

$\mathrm{S}-1$ is an oral fluoropyrimidine that consists of tegafur, which is a 5-FU prodrug, combined with gimeracil and oteracil potassium. Gimeracil (5-chloro-2, 4-dihydroxypyridine) acts as a potent inhibitor of 5-FU degradation, and oteracil potassium reduces the gastrointestinal toxicity caused by 5-FU (11). S-1 monotherapy is considered as the second- or third-line chemotherapy for unresectable and recurrent esophageal squamous cell carcinoma (12). While S-1 monotherapy is safe and well tolerated in elderly patients, it is also associated with modest response rates and infrequent responses (13). However, several reported cases from Japan have shown a marked response to S-1/CDDP chemotherapy in patients with recurrent esophageal cancer $(14,15)$.

In a previous study, we reported a CR in a patient who presented with advanced esophageal cancer and abdominal bulky lymph node metastasis, and was treated with concurrent chemoradiotherapy using docetaxel, CDDP and 5-fluorouracil (16). Although S-1 administration alone is not considered to exhibit a significant effect on recurrent lesions, the treatment in the present study showed unexpected effectiveness. Overall, the reported therapy may be considered as an option for the treatment of patients with a poor performance status and locally advanced, recurrent or metastatic esophageal cancer.

\section{References}

1. Pennathur A, Gibson MK, Jobe BA and Luketich JD: Oesophageal carcinoma. Lancet 381: 400-412, 2013.

2. Zhang Y: Epidemiology of esophageal cancer. World J Gastroenterol 19: 5598-5606, 2013.

3. Enzinger PC and Mayer RJ: Esophageal Cancer. N Engl J Med 349: 2241-2252, 2003.

4. Quint LE, Hepburn LM, Francis IR, Whyte RI and Orringer MB: Incidence and distribution of distant metastases from newly diagnosed esophageal carcinoma. Cancer 76: 1120-1125, 1995.

5. Ilson DH: Esophageal cancer chemotherapy: Recent advances. Gastrointest Cancer Res 2: 85-92, 2008.
6. Greene FL, Page DL, Fleming ID, Fritz AG, Balch CM, Haller DG and Morrow M (eds): Esophagus. In: American Joint Committee on Cancer (AJCC) Cancer Staging Manual. 6th edition. Springer, New York, NY, pp90-95, 2002.

7. Trotti A, Colevas AD, Setser A, Rusch V, Jaques D, Budach V, Langer C, Murphy B, Cumberlin R, Coleman CN and Rubin P: CTCAE v3.0: Development of a comprehensive grading system for the adverse effects of cancer treatment. Semin Radiat Oncol 13: 176-181, 2003.

8. Iizuka T, Kakegawa T, Ide H, Ando N, Watanabe H, Tanaka O, Takagi I, Isono K, Ishida K, Arimori M, et al: Phase II evaluation of cisplatin and 5-fluorouracil in advanced squamous cell carcinoma of the esophagus: A Japanese esophageal oncology group trial. Jpn J Clin Oncol 22: 172-176, 1992.

9. The National Comprehensive Cancer Network (NCCN) Guidelines Version 1.2014: Esophageal and Esophagogastric Junction Cancers. http://www.nccn.org/professionals/physician_ gls/pdf/esophageal.pdf. Accessed March 1, 2015.

10. Cunningham D, Okines AF and Ashley S: Capecitabine and oxaliplatin for advanced esophagogastric cancer. N Engl J Med 362: 858-859, 2010.

11. Shirasaka T, Shimamato Y, Ohshimo H, Yamaguchi M, Kato T, Yonekura K and Fukushima M: Development of a novel form of an oral 5-fluorouracil derivative (S-1) directed to the potentiation of the tumor selective cytotoxicity of 5-fluorouracil by two biochemical modulators. Anticancer Drugs 7: 548-557, 1996.

12. Akutsu Y, Kono T, Uesato M, Hoshino I, Narushima K, Hanaoka T, Tochigi T, Semba Y, Qin W and Matsubara H: S-1 monotherapy as second- or third-line chemotherapy for unresectable and recurrent esophageal squamous cell carcinoma. Oncology 84: 305-310, 2013.

13. Koizumi W, Narahara H, Hara T, Takagane A, Akiya T, Takagi M, Miyashita K, Nishizaki T, Kobayashi O, Takiyama W, et al: S-1 plus cisplatin versus $\mathrm{S}-1$ alone for first-line treatment of advanced gastric cancer (SPIRITS trial): A phase III trial. Lancet Oncol 9: 215-221, 2008.

14. Hiraki M, Yunotani S, Noguchi R, Shinozaki Y, Tani H, Sakai M, Ishimitsu $\mathrm{T}$ and Tabuchi M: Recurrence of esophageal cancer treated by combination TS-1/CDDP therapy. Gan To Kagaku Ryoho 32: 219-221, 2005 (In Japanese).

15. Kanamori N, Fujii M, Takahashi T, Wakabayashi K, Kochi M, Sou K and Takayama T: A patient with esophageal cancer recurrence responding to $\mathrm{S}-1$ combined with cisplatin (CDDP). Gan To Kagaku Ryoho 34: 1459-1461, 2007 (In Japanese).

16. Kubota K, Mafune K, Yamada K, Yamashita H, Kuroda J, Aikou S and Kaminishi M: Complete regression of advanced esophageal cancer with abdominal bulky lymph node metastasis treated by concurrent chemoradiotherapy using docetaxel, cisplatin and 5-fluorouracil. Esophagus 6: 183-187, 2009. 